\title{
A computer program for measuring body size distortion and body dissatisfaction
}

\author{
RICK M. GARDNER and RUSSEL BOICE \\ University of Colorado, Denver, Colorado
}

\begin{abstract}
A computer program is described that measures body size distortion and body dissatisfaction. The program is written using Visual Basic development tools and will run on any Windows 98 or more current system. The width of a static digital image of the participant can be manipulated using three separate psychophysical methods. In the method of adjustment, the participant adjusts the image wider or thinner to match his or her perceived size. The participants may also be required to adjust the image to their ideal size, with the discrepancy between perceived and ideal size being used as a measure of body dissatisfaction. In the staircase method, participants see an image that is continuously expanding or contracting. The participants change the direction of the distortion when the image matches their perceived size. In the adaptive probit estimation procedure, participants judge whether a static image is distorted too wide or too thin. Analysis of the responses permits a determination of the point of subjective equality (PSE) and the difference limen (DL) values. The DL reflects the amount of body size distortion necessary for the participant to detect the distortion $50 \%$ of the time. The PSE reflects the participant's subjective judgment of his or her body size. These two values are reflective of the sensory and nonsensory components, respectively, that contribute to body size judgments.
\end{abstract}

There has been an increasing interest in the topic of body image in recent years. Research has shown a clear link between how an individual sees himself or herself and several forms of pathology, especially eating disorders. In addition, numerous studies have linked body image disturbance to low self-esteem, neuroticism, depression, and general psychological distress.

Researchers distinguish between two separate components of body image disturbance-namely, perceptual body size distortion and the attitudinal, or affective, element. Perceptual distortion consists of inaccurate judgments of one's body size. For example, individuals with eating disorders frequently overestimate their actual body size. The attitudinal component consists of dissatisfaction with one's body size, shape, or some other aspect of body appearance (Gardner, 2001).

Researchers in eating disorders have predominantly used the perceptual assessment, whereas measures of the attitudinal component have been used less frequently. Interestingly, the perceptual and attitudinal components appear to function largely independently of one another (Garner \& Garfinkel, 1981). Researchers have increasingly come to believe that body image is a multidimen-

Correspondence concerning this article should be addressed to R. M. Gardner, Department of Psychology, University of Colorado at Denver, P. O. Box 173364, Campus Box 173, Denver, CO 80217-3364 (e-mail: rgardner@carbon.cudenver.edu).

Note-The program is available on request by e-mail at the address above. Users who adopt the program will be asked to make a contribution toward its development costs. sional construct and that the assessment of body image disturbance requires multimethod and multimodal measurement techniques (Keeton, Cash, \& Brown, 1990).

A variety of techniques have been developed to measure the attitudinal component of body image disturbance. Body dissatisfaction is often measured with schematic drawings or figural scales. These scales, sometimes referred to as silhouettes, consist of a set of line drawings of the human body that range in size from very underweight to very overweight. Participants select the drawing that they feel corresponds to their actual body size, as well as to the size they would like to be ideally. The discrepancy between these two measures reflects body dissatisfaction. One limitation of these types of scales is that they measure only the affective component of body image disturbance and do not give any measure of perceptual size distortion. In addition, these scales have numerous inherent methodological deficiencies that limit both their reliability and their validity (Gardner, Friedman, \& Jackson, 1998).

Several innovative techniques have been developed to measure the perceptual component of body image disturbance (see Gardner, 2001, and Thompson, 1996, for reviews). Some techniques involve whole-image procedures that adjust the body size smaller or larger via modification of a photograph or a video image. In recent years, investigators have increasingly turned to video techniques to measure the extent of body size distortion (Thompson \& Smolak, 2001). Although there have been several variants of this procedure, all consist of presenting a static video image of a participant's body. The participant adjusts the width of the image while the height remains constant. Most investigators have used this procedure to distort 
whole images of the body, although the procedure has also been used to estimate the size of specific body sites (Gardner \& Bokenkamp, 1996). The video methodology has been used successfully with children as young as 5 years of age (Gardner, Gardner, \& Morrell, 1990).

Nearly all the investigators using the video technique have employed the psychophysical method of adjustment. In this method, the participant is presented a static image of his or her body, either shown on a TV monitor or projected life size on a screen, which is initially distorted to be either too large or too small. The participant is required to adjust the width of the image to match his or her perceived body size. Several trials are used, including ascending trials in which the image is initially distorted so as to be too thin and descending trials in which the initial image is distorted so as to be too wide. A body perception index is derived that reflects the direction and degree of size distortion, relative to actual body size. In addition, participants are often asked to adjust the width of the image to the size they would like to be ideally. The discrepancy between the perceived and the ideal body sizes is commonly used as a measure of body dissatisfaction.

Gardner (1996) has noted several methodological issues that are important in the assessment of the perceptual component of body image disturbance. When the participant uses the method of adjustment, the initial body size that is observed serves as an anchor that greatly influences the final size judgment (Probst, Van Coppenolle, Vandereycken, \& Goris, 1992). Several investigators have consistently found that for those participants who are asked to decrease an initial image that is too wide, the image that represents their final judgment of their body will be too wide and that for those who are asked to increase an image that is too thin, the image that represents their final judgment will be too thin. Typically, investigators take an average of the ascending and descending trials to find an "average" body size judgment. Gardner (1996) notes that taking an average of these ascending and descending trials results in an average body size judgment that does not accurately represent either the ascending or the descending trials. Compounding the problem, Gardner and his colleagues have consistently found that the error of anticipation is not the same for ascending and descending trials, with estimates closer to actual body size occurring on ascending, as compared with descending, trials (Gardner, 1996). This asymmetry can serve to invalidate the common practice of averaging across ascending and descending trials to determine a participant's body size estimate.

The early psychophysicists noted in their original work with the method of adjustment that nonsensory factors, such as participants' attitudes, motivations, expectations, and so forth, regularly influence their judgments about aspects of a stimulus (Gescheider, 1976). The early researchers attempted to control for these nonsensory factors by using highly trained and experienced participants.

One way to avoid the error of anticipation problems is to employ some variant of the method of constant stimuli, wherein ascending/descending trials are not used. In this procedure, a range of static stimuli are presented repeatedly throughout the experiment. For body size estimations, the participant is presented a range of stimuli from those well below his or her actual size to those well above his or her actual size. On a given trial, one of these distortions is presented, and the participant must report whether it represents an over- or an underestimation. The resulting psychophysical function reveals the typical ogive (sigmoid) shape, with body size estimation (referred to as the point of subjective equality, or PSE) being that size where the participant reported $50 \%$ of the images as overestimations and $50 \%$ as underestimations. In addition, this procedure allows one to ascertain how much distortion in body size beyond this PSE is necessary before the person detects the change as a just noticeable difference (JND). This value is referred to as the difference threshold, or difference limen (DL). Although the method of constant stimuli has been used successfully to estimate body size perceptions (Gardner, Morrell, Watson, \& Sandoval, 1989), it has the inherent disadvantage of requiring the presentation of a large number of trials at several different stimulus values and, therefore, is time consuming and burdensome for the participants (Gardner, 1996).

Recently, Gardner and his colleagues have used an advanced hybrid psychophysical technique called adaptive probit estimation (APE), which estimates a complete psychometric function with maximum statistical efficiency while using minimal labor by participants (Gardner, Jones, \& Bokenkamp, 1995). Based on the method of constant stimuli, APE requires that participants make a forced choice between two alternatives (image too wide or too thin) in response to an amount of body size distortion. It does not, however, require the lengthy number of trials inherent in the method of constant stimuli.

A complete discussion of APE is beyond the scope of this article, and the reader is referred to Watt and Andrews (1981) for a detailed explanation. Briefly, the experimenter estimates the mean and standard deviation of the error distribution for body size estimates and presents four stimulus distortion levels at \pm 0.45 and $\pm 1.35 z$ values. For example, assume that a PSE of $0 \%$ body size distortion will be anticipated. Previous research by Gardner and his colleagues has indicated that the standard deviation of obtained distortion values for most participants falls at or below $7.27 \%$. Given these parameters, a participant's video image was presented at four levels of distortion: $9.81 \%$ too wide, $3.27 \%$ too wide, $3.27 \%$ too thin, and $9.81 \%$ too thin. Eight blocks of 10 presentations of each of the four distortion levels were used. The participant was required to judge whether each image was too wide or too thin. Figure 1 illustrates the percentage of larger than responses to the four levels of body size distortion one would anticipate and illustrates these values plotted with a best fit cumulative normal sigmoid function (from Gardner, 1996). As with the method of constant stimuli, the best fit psychophysical function reveals simultaneously the PSE and the difference threshold. In this example, the participant would have responded that an image distorted 


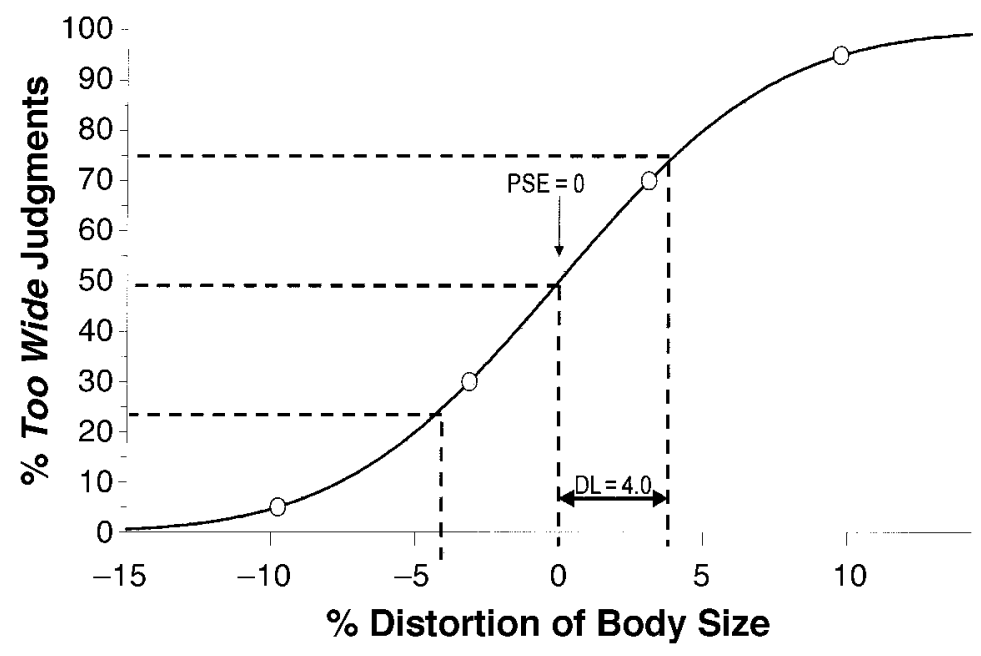

Figure 1. The best fit cumulative normal sigmoid function for too wide judgments presented at four levels of distortion. The point of subjective equality (PSE) corresponds to that percentage of body size distortion at which the participant made $50 \%$ too wide judgments. The difference threshold (difference limen, DL) reflects the amount of change in body size distortion necessary for the participant to detect a change in body size $50 \%$ of the time. Reproduced from Gardner (1996), with permission from the British Journal of Psychology; copyright 1996, the British Psychological Society.

$0 \%$ would have appeared wider than their actual body size $50 \%$ of the time, thus indicating a PSE of $0 \%$. The standard deviation of the obtained wider than percentages about the $50 \%$ point indicates the slope of the psychophysical function. A larger slope (and correspondingly, a smaller standard deviation) indicates a greater sensitivity of the participant to detect changes in stimulus size. The DL corresponds to the amount of change in body size necessary for the participant to detect the change $50 \%$ of the time and is illustrated in Figure 1 as those distortion levels corresponding to $25 \%$ and $75 \%$ too wide judgments. Thus, the standard deviation is a measure of the participant's sensitivity in detecting changes in body size and is a relatively unbiased indicator of sensory factors. For the data illustrated in Figure 1, the PSE equals 0\% distortion with a DL of $4.0 \%$.

As Gardner (1996) noted, the adaptive probit estimation procedure can be seen to mimic closely the procedure used with the method of constant stimuli. Where it differs is that the four stimulus levels are subject to revision. At the end of the second and every subsequent block, a rapid probit analysis and computation of the PSE and DL for the previous two blocks is made. The four stimulus levels are then reselected as necessary, on the basis of this analysis (Watt \& Andrews, 1981). If the PSE obtained in the first two blocks is found to be not centered in the four stimulus presentation values, an appropriate adjustment is made in the subsequent four distortion levels, which are presented in the next block of trials. Similarly, if the four distortion levels represented a task that was too easy or too difficult for the participant, the standard deviation of the subsequent distortion values is adjusted to make the difficulty of the discrimination more appropriate on the subsequent block. Only a partial correction of the PSE and standard deviation to the stimulus test set is made, since a full correction makes the system too sensitive to transient changes in the participant's PSE. The first two blocks use predetermined parameters for the distortion levels presented. Starting with the third block, both the mean and the magnitude of the four levels of distortion presented are adjusted. The adjustment applied to the third block is based on the average results of the analysis of the first two blocks. For all subsequent blocks, the adjustment factors are based on the analysis of each previous block. In each case, the amount of the adjustment made to the distortion levels presented is one half of that which would be expected to result in the target response ratios. The application of one half of the full correction, rather than using the full amount of correction in distortion levels for adjusted blocks, serves to prevent the oscillation in the block-byblock distortion parameters that otherwise occurs in practice (see Watt \& Andrews, 1981).

After a series of such blocks, an average PSE and rootmean square standard deviation are calculated to reflect overall performance.

\section{BODY SIZE DISTORTION PROGRAM}

The present computer program allows the investigator a choice of three psychophysical methods: the method of adjustment, the staircase method (a derivative of the method of adjustment), and the APE technique. The program is written using Visual Basic development tools and will run on any Windows 98 or more current system with 
VGA or better resolution. The program operates most efficiently with a Pentium III or faster processor. The program requires the input of a static digital image. Such images are easily obtained with a digital camera. Most investigators use an image of the full frontal profile, although any perspective can be used.

\section{Method of Adjustment}

Although the method of adjustment has certain limitations, as was noted earlier, it is still the most commonly used procedure for testing body size distortion with a video technique. In the present program, a digitally stored image of the participant is initially presented at distortion levels ranging between $\pm 20 \%$ and $\pm 30 \%$ too wide or too thin. The participant can then adjust the width of the image by pressing the right or the left button on a mouse. Holding down the right button on the mouse causes the displayed image's actual width to expand wider at the rate of $1 \%$ per second, whereas the left button causes the displayed width to decrease at the same rate. Participants are typically instructed to make as many adjustments as necessary until they believe that the image is an accurate representation of their actual body size. The participants depress the middle button or scroll wheel when they are satisfied that the adjusted image is the accurate size. The investigator is able to control the number of data collection trials, with the default value set to 10 .

This method can also be used to determine the body size participants wish to be ideally. Participants are required to adjust the initially distorted image wider or thinner until it reaches their desired ideal size. As was noted earlier, the resulting discrepancy between actual perceived size and ideal size is commonly used as a measure of body dissatisfaction.

Table 1 illustrates sample data output for the method of adjustment. The output includes the percentage of initial distortion present at the beginning of each trial, the amount of ending distortion present after the participant has adjusted the width of the image, the time in seconds the participant took in making the adjustment, and the number of adjustments, wider and thinner, the participant made in arriving at the final judgment. Finally, an average and a standard deviation are given for each of these values for ascending trials, descending trials, and all trials combined. By illustrating the values separately for ascending and descending trials, the investigator can readily detect the magnitude of any errors of anticipation associated with the ascending and descending trials. Most investigators will likely use the overall average distortion as the best single measure of body size distortion with this method, although the limitations of doing so were discussed earlier.

For the sample data shown, the participant viewed an image that was initially presented as $21.72 \%$ too thin on Trial 1. The participant adjusted the image on this trial to an overestimation of $0.25 \%$, taking $29.84 \mathrm{sec}$ while making one wider/thinner adjustment. Summary data at the bottom of Table 1 illustrate that the average distortion made on descending trials was a $5.04 \%$ overestimation $(s=2.04)$, whereas on ascending trials the participant overestimated by $7.61 \%(s=6.32)$. Overall average distortion, including both ascending and descending trials, was an overestimation of $6.32 \%(s=4.63)$. Summary data for the time required to reach final judgments and the number of wider/thinner adjustments made are also illustrated. The time required to reach final adjustment includes the time from when the initial image was presented until the participant pressed the center mouse button and thus includes time before, between, and after buttonpresses. Participants' data from this and each of the following two methodologies can be found in a Word file called cojdata.doc, located in My Documents.

\section{Staircase Method}

The staircase method (Cornsweet, 1962) is a variation of the psychophysical method of limits. Participants are

Table 1

Sample Output Using the Method of Adjustment

\begin{tabular}{|c|c|c|c|c|c|c|c|}
\hline $\begin{array}{c}\text { Trial } \\
\text { Number }\end{array}$ & $\begin{array}{c}\text { Initial \% } \\
\text { Distortion }\end{array}$ & $\begin{array}{c}\text { End \% } \\
\text { Distortion }\end{array}$ & & $\begin{array}{l}\text { Time } \\
(\mathrm{sec})\end{array}$ & \multicolumn{3}{|c|}{$\begin{array}{c}\text { Number of } \\
\text { Adjustments }\end{array}$} \\
\hline 1 & -21.72 & 0.25 & & 29.84 & \multicolumn{3}{|c|}{1} \\
\hline 2 & 20.14 & 4.84 & & 19.36 & \multicolumn{3}{|c|}{2} \\
\hline 3 & -21.63 & 3.80 & & 36.02 & \multicolumn{3}{|c|}{3} \\
\hline 4 & 28.14 & 3.73 & & 27.47 & \multicolumn{3}{|c|}{4} \\
\hline 5 & -21.32 & 6.86 & & 35.84 & \multicolumn{3}{|c|}{2} \\
\hline 6 & 20.45 & 4.33 & & 17.03 & \multicolumn{3}{|c|}{1} \\
\hline 7 & 24.14 & 3.70 & & 28.94 & \multicolumn{3}{|c|}{4} \\
\hline 8 & -22.26 & 10.44 & & 39.41 & \multicolumn{3}{|c|}{2} \\
\hline 9 & 27.90 & 8.58 & & 32.66 & \multirow{2}{*}{\multicolumn{3}{|c|}{$\begin{array}{l}5 \\
1\end{array}$}} \\
\hline \multirow[t]{2}{*}{10} & -19.18 & 16.69 & & 39.02 & & & \\
\hline & & $\begin{array}{c}\text { Average } \\
\text { Distortion } \\
\end{array}$ & $S D$ & $\begin{array}{l}\text { Time } \\
(\mathrm{sec})\end{array}$ & $S D$ & $\begin{array}{c}\text { Average } \\
\text { Adjustments }\end{array}$ & $S D$ \\
\hline \multicolumn{2}{|c|}{ Decending Trials } & 5.04 & 2.04 & 25.09 & 6.62 & 3.20 & 1.64 \\
\hline \multirow{2}{*}{\multicolumn{2}{|c|}{$\begin{array}{l}\text { Ascending Trials } \\
\text { Overall }\end{array}$}} & 7.61 & 6.32 & 36.02 & 3.83 & 1.80 & 0.84 \\
\hline & & 6.32 & 4.63 & 30.56 & 7.70 & 2.50 & 1.43 \\
\hline
\end{tabular}


initially presented with an image that is an accurate representation of their body size. Immediately after appearing, the displayed image's width is sequentially increased at a rate of $1 \%$ per second. Participants are instructed to press the right button on the hand-held mouse when the body size is the same as their perceived actual size. The direction of distortion is then reversed, and the participant is instructed to press the left button when the image, now being sequentially distorted in the opposite direction, has become equal to their perceived size. These wider and thinner sequences continue until data collection is complete. The number of wider/thinner sequences is adjustable, with the default number set at 10 . The computer records the percentage of overestimation and/or underestimation at each direction change. Overall, perceived body size is taken as the average of the transition points. This method allows for very rapid data collection over even a large number of trials. The fast-paced nature of the task is especially helpful when younger children are tested (Gardner, 2001).

Table 2 illustrates the data output from the staircase method. Note that on Trial 1, the participant reversed the increasingly thinner image when it reached $4.858 \%$ too small, again on the next expanding trial, when the image was $0.752 \%$ too small, and so forth for the continuing trials. The average of the transition points is shown at the bottom of the output to be $-2.191 \%$, which shows that, overall, the participant underestimated the body width by $2.191 \%$, with a standard deviation of 2.198 .

\section{Adaptive Probit Estimation (APE)}

As was noted earlier, the APE procedure is a derivative of the psychophysical method of constant stimuli. A static image of the participant is initially presented at four levels of distortion: $\pm 9.81 \%$ too wide and too thin and $\pm 3.27 \%$ too wide and too thin. Eight blocks of 10 presentations of each of the four distortion levels is presented, with the participant judging whether each image is too wide or too thin. The number of blocks to be used is adjustable by the experimenter. Beginning with the third block of trials, an analysis is made of the participant's PSE and DL. Following the first two blocks, the amount of dis-

Table 2

Sample Output Using the Staircase Method

\begin{tabular}{cc}
\hline Trial & \% Distortion \\
\hline 1 & -4.858 \\
2 & -0.752 \\
3 & -4.336 \\
4 & -0.501 \\
5 & 0.752 \\
6 & -2.785 \\
7 & 0.250 \\
8 & -3.557 \\
9 & -1.004 \\
10 & -5.121 \\
Average & -2.191 \\
$S D$ & 2.198 \\
\hline
\end{tabular}

tortion of the four stimulus presentation values is adjusted in each subsequent block to approximately center the participant's PSE in the four levels of distortion. Similarly, the standard deviation of the presented stimuli is adjusted to continue to ensure that the discrimination task is moderately difficult for the subsequent block. As was described earlier, some inertia is programmed into these corrections to prevent oscillation in participants' judgments.

Table 3 illustrates a sample output for the APE technique. The first column shows the four distortion levels used in the initial two blocks of presentations. Note that the four distortion levels remain constant throughout the first two blocks of trials and then change in subsequent blocks to keep the four levels approximately centered about the participant's PSE. The second column, labeled "Response Rate," shows the proportion of too wide judgments made at each level of distortion.

The third column is labeled "Standard Deviation" and shows the amount of variation used in the four distortion levels. This amount remains constant at $7.27 \%$ for the first two blocks and then is automatically adjusted to maintain a moderately difficult discrimination task. For this sample data, the participant's level of accuracy was such that in Block 3, the program made the discrimination slightly more difficult by reducing the standard deviation of the four stimulus levels to 5.14. Similar adjustments were made for each of the remaining blocks.

The column labeled "PSE Offset" shows the amount of adjustment necessary about the center of the four distortion levels to keep the participant's PSE in the approximate center of the four distortion levels. Note that no offset is used for the first two blocks. Beginning in the third block, the sample output shows that a slight adjustment of $1.22 \%$ was required to accomplish this centering. It is the combination of the adjusted standard deviation and the PSE that results in the revised set of distortion levels present in Block 3 and subsequent blocks.

The column labeled "Slope" shows the slope of the best fit line of the cumulative normal sigmoid function. The slope is that of the line computed on the basis of finding the sigmoid least squares fit for the four points, with the target ratio as the $x$-coordinate and the measured response ratio for the $y$-coordinate. This line also determines the $y$-intercept.

Recall that a larger slope indicates a greater sensitivity of the participant in detecting changes in stimulus size. Note that beginning with Block 3, as the slope becomes slightly smaller, the standard deviation also becomes smaller, illustrating the relative difficulty of the discrimination task. The next column, labeled " $y$-intercept" shows the intercept of the best fit line.

The last two columns reflect the data that most experimenters will be interested in. The column labeled "DL" shows the DL for each block of trials. Recall that the DL, also sometimes called the difference threshold, is the amount of change in body size necessary for the participant to detect that change $50 \%$ of the time. It is a measure of the participant's sensitivity in detecting changes in body size. Gardner (2001) details how the DL is a relatively un- 
Table 3

Sample Output Using the Adaptive Probit Estimation (APE) Method

\begin{tabular}{|c|c|c|c|c|c|c|c|c|}
\hline & $\begin{array}{l}\text { Distortion } \\
\text { Level }(\%)\end{array}$ & $\begin{array}{l}\text { Response } \\
\text { Rate }\end{array}$ & $S D$ & $\begin{array}{c}\text { PSE } \\
\text { Offset }\end{array}$ & Slope & $y$-Intercept & DL & PSE \\
\hline Block 1 & $\begin{array}{r}-9.81 \\
-3.27 \\
3.27 \\
9.81\end{array}$ & $\begin{array}{l}0.0 \\
0.2 \\
0.7 \\
1.0\end{array}$ & 7.27 & 0.00 & 1.58 & -0.16 & 3.11 & 0.73 \\
\hline Block 2 & $\begin{array}{r}-9.81 \\
-3.27 \\
3.27 \\
9.81\end{array}$ & $\begin{array}{l}0.1 \\
0.0 \\
0.7 \\
1.0\end{array}$ & 7.27 & 0.00 & 2.57 & -0.60 & 1.91 & 1.70 \\
\hline Block 3 & $\begin{array}{r}-8.86 \\
-3.15 \\
2.55 \\
8.26\end{array}$ & $\begin{array}{l}0.0 \\
0.1 \\
0.8 \\
1.0\end{array}$ & 5.14 & 1.22 & 2.36 & -0.22 & 1.47 & 1.70 \\
\hline Block 4 & $\begin{array}{r}-8.50 \\
-3.26 \\
1.97 \\
7.20\end{array}$ & $\begin{array}{l}0.4 \\
0.7 \\
0.8 \\
1.0\end{array}$ & 3.64 & 1.70 & 0.89 & 0.97 & 2.75 & -2.27 \\
\hline Block 5 & $\begin{array}{r}-7.61 \\
-3.13 \\
1.35 \\
5.83\end{array}$ & $\begin{array}{l}0.2 \\
0.3 \\
0.9 \\
0.9\end{array}$ & 3.85 & -0.29 & 1.22 & 0.31 & 2.13 & -1.25 \\
\hline Block 6 & $\begin{array}{r}-7.16 \\
-2.61 \\
1.93 \\
6.48\end{array}$ & $\begin{array}{l}0.0 \\
0.2 \\
0.3 \\
1.0\end{array}$ & 3.48 & -1.76 & 2.31 & -1.47 & 1.02 & 0.46 \\
\hline Block 7 & $\begin{array}{r}-7.16 \\
-2.61 \\
1.94 \\
6.48\end{array}$ & $\begin{array}{l}0.2 \\
0.5 \\
0.9 \\
1.0\end{array}$ & 2.46 & -0.40 & 1.16 & 0.61 & 1.44 & -1.70 \\
\hline Block 8 & $\begin{array}{r}-6.86 \\
-2.76 \\
1.33 \\
5.43\end{array}$ & $\begin{array}{l}0.1 \\
0.6 \\
0.3 \\
0.7\end{array}$ & 2.29 & -0.62 & 0.48 & -0.42 & 3.22 & 1.38 \\
\hline \multicolumn{9}{|c|}{$\begin{array}{l}\text { Mean DL }(\text { root-mean square })=2.265 \\
\text { Mean PSE }=0.095\end{array}$} \\
\hline
\end{tabular}

biased indicator of sensory factors. Note that for Block 1, the participant required a change in body size of $3.11 \%$ to detect the change $50 \%$ of the time. Sensory sensitivity should remain relatively stable throughout the eight blocks, which is observed in the sample data. The last column is labeled "PSE" and reflects the participant's PSE. Again, this is the amount of distortion necessary for the participant to report $50 \%$ of the images as overestimations and $50 \%$ as underestimations. As such, the PSE reflects the nonsensory, or affective, components of body image judgments. In the sample data, the participant's PSE for the first block was $0.73 \%$, which can be interpreted as meaning that the participant judged that a body size that was $0.73 \%$ too wide was subjectively equal to what the participant judged the size to actually be. The PSE is a measure of body size judgments that is uncontaminated by sensory sensitivity factors (Gardner, 2001). That is, the PSE and the DL are independent measures of different aspects of a participant's perceived body image.

Summary data for both the DL and the PSE are illustrated at the bottom of Table 3 . The mean DL is the root- mean square of the DL values obtained in each of the eight blocks of trials. The mean PSE is the average of the PSE values obtained in each of the eight blocks. These two summary values are the best overall indicators of the participant's over/underestimation of body size, as well as their sensory sensitivity to detecting body size distortion.

Gardner and his colleagues have demonstrated the efficacy of these three psychophysical methods in several studies with participants of varying gender, ethnicity, and ages, as well as with participants who have eating disorders (Gardner \& Bokenkamp, 1996; Gardner, Espinoza, Urrutia, Morrell, \& Gallegos, 1989; Gardner, Friedman, \& Jackson, 1999a, 1996b; Gardner, Friedman, Stark, \& Jackson, 1999; Gardner, Gallegos, Martinez, \& Espinoza, 1989; Gardner, Gardner, \& Morrell, 1990; Gardner, Jones, \& Bokenkamp, 1995; Gardner, Morell, Urrutia, \& Espinoza, 1989; Gardner, Morrell, Watson, \& Sandoval, 1990; Gardner, Sorter, \& Friedman, 1997; Gardner, Stark, Friedman, \& Jackson, 2000; Gardner \& Tockerman, 1993). The ability of other researchers to use these psychophysical techniques has been limited by the lack of 
availability of software programs that will run on unmodified personal computers. The complexity of the APE technique requires such a computer program in order to present the appropriate stimuli.

Shibata (2002) has recently developed a computer program for assessing body image disturbance that uses only the method of adjustment. The present program adds two other psychophysical methods to measure body size distortion and dissatisfaction. It is the only program that allows use of the APE methodology. It requires only a standard personal computer (preferably with a Pentium III or better processor) and a digital camera for implementation and should prove useful to investigators interested in gaining more technical and methodological sophistication in measuring aspects of body size perception.

Finally, instructors who are teaching students about psychophysical methods should find this program useful. The program readily allows students to evaluate judgments, using three psychophysical methods.

\section{Availability}

The copyrighted program may be obtained as an e-mail attachment from the first author at rgardner@carbon. cudenver.edu or on disk by requesting the same from R. M. Gardner, Department of Psychology, University of Colorado at Denver, P. O. Box 173364, Denver, CO $80217-$ 3364.

\section{REFERENCES}

CoRnsweEt, T. N. (1962). The staircase method in psychophysics. American Journal of Psychology, 75, 485-568.

GARDNER, R. M. (1996). Methodological issues in assessment of the perceptual component of body image disturbance. British Journal of Psychology, 87, 327-337.

GARDNER, R. M. (2001). Assessing body image disturbance in children and adolescents. In J. K. Thompson and L. Smolak (Eds.), Body image, eating disorders, and obesity in children and adolescents: Theory, assessment, treatment, and prevention (pp. 193-214). Washington DC: American psychological Association.

GARDNER, R. M., \& BoKEnKAMP, E. D. (1996). The role of sensory and non-sensory factors in body estimations of eating disorder subjects. Journal of Clinical Psychology, 52, 3-15.

Gardner, R. M., Espinoza, T., Urrutia, R., Morrell, J., \& GalleGOS, V. (1989). Effect of hunger on body image in the obese: Feeling full, feeling fat. Psychiatric Forum, 15, 57-62.

GARdner, R. M., Friedman, B. N., \& JaCKson, N. A. (1998). Methodological concerns when using silhouettes to measure body image. Perceptual \& Motor Skills, 86, 387-395.

Gardner, R. M., Friedman, B. N., \& JaCkson, N. A. (1999a). Body size estimations, body dissatisfaction, and ideal size preferences in children six through thirteen. Journal of Youth \& Adolescence, 28, 603-618.

GARdNER, R. M., Friedman, B. N., \& JACKSON, N. A. (1999b). Gender and ethnic differences in children's judgments of perceived and ideal body size in self and others. Psychological Record, 49, 555-564.
Gardner, R. M., Friedman, B. N., Stark, K., \& Jackson, N. A. (1999). Body size estimations in children six through fourteen: A longitudinal study. Perceptual \& Motor Skills, 88, 541-545.

Gardner, R. M., Gallegos, V., Martinez, R., \& Espinoza, T. (1989). Mirror feedback and body image: A psychophysical approach. Journal of Psychosomatic Research, 33, 603-607.

Gardner, R. M., Gardner, E. A., \& Morrell, J. A. (1990). Body image of sexually and physically abused children. Journal of Psychiatric Research, 24, 313-321.

GARDNER, R. M., JonES, L., \& BoKenKAMP, E. (1995). Adaptive probit estimation of body size: A comparison of three psychophysical techniques. Perceptual \& Motor Skills, 80, 1379-1390.

Gardner, R. M., Morrell, J. [A.], Urrutia, R., \& Espinoza, T. (1989). Body image following significant weight loss. Journal of Personality \& Social Behavior, 4, 603-614.

Gardner, R. M., Morrell, J. A., Watson, D. N., \& Sandoval, S. L. (1989). Subjective equality and just noticeable differences in body size judgments by obese persons. Perceptual \& Motor Skills, 69, 595604.

Gardner, R. M., Morrell, J. A., Watson, D. N., \& Sandoval, S. L. (1990). Eye movements and body size judgments in the obese. International Journal of Eating Disorders, 9, 537-544.

Gardner, R. M., Sorter, R., \& Friedman, B. N. (1997). Developmental changes in children's body image. Journal of Social Behavior \& Personality, 12, 1019-1036.

Gardner, R. M., Stark, K., Friedman, B. N., \& Jackson, N. A. (2000). Predictors of eating disorders scores in children ages six through fourteen: A longitudinal study. Journal of Psychosomatic Research, 49, 199-205.

GARDNER, R. M., \& TOCKERMAN, Y. (1993). Body dissatisfaction as a predictor of body size distortion: A multidimensional analysis of body image. Genetic, Social, \& General Psychology Monographs, 199, 127-145.

GARNER, D., \& GARFINKEL, P. E. (1981). Body image in anorexia nervosa: Measurement, theory and clinical implications. International Journal of Psychiatric Medicine, 11, 263-284.

Gescheider, G. A. (1976). Psychophysics: Method and theory. Hillsdale, NJ: Erlbaum.

Keeton, W. P., CASH, T. F., \& Brown, T. A. (1990). Body image or body images? Comparative, multidimensional assessment among college students. Journal of Personality Assessment, 54, 213-230.

Probst, M., Van Coppenolle, H., Vandereycken, W., \& Goris, M. (1992). Body image assessment in anorexia nervosa patients and university students by means of video distortion: A reliability study. Journal of Psychosomatic Research, 36, 89-97.

ShiBATA, S. (2002). A Macintosh and Windows program for assessing body-image disturbance using adjustable image distortion. Behavior Research Methods, Instruments, \& Computers, 34, 90-92.

Thompson, J. K. (1996). Assessing body image disturbance: Measures, methodology, and implementation. In J. K. Thompson (Ed.), Body image, eating disorders, and obesity (pp. 49-81). Washington, DC: American Psychological Association.

Thompson, J. K., \& SMOLAK, L. (Eds.) (2001). Body image, eating disorders, and obesity in youth: Assessment, prevention, and treatment. Washington, DC: American Psychological Association.

WATT, R. J., \& ANDREWS, D. P. (1981). APE: Adaptive probit estimation of psychometric functions. Current Psychology Review, 1, 205-214.

(Manuscript received June 30, 2003; accepted for publication July 22, 2003.) 